\title{
The influence of the amplitude of low-frequency fluctuations on resting-state functional connectivity
}

\author{
Xin Di ${ }^{1}$, Eun H. Kim ${ }^{1}$, Chu-Chung Huang ${ }^{2}$, Shih-Jen Tsai ${ }^{2}$, Ching-Po Lin ${ }^{2}$ and Bharat B. Biswal ${ }^{1}{ }^{*}$
}

1 Department of Biomedical Engineering, New Jersey Institute of Technology, Newark, NJ, USA

2 Institute of Neuroscience, National Yang-Ming University, Taipei, Taiwan

\section{Edited by:}

Veronika Schöpf, Medical University

Vienna, Austria

\section{Reviewed by:}

Ahmed Abou Elseoud, Oulu University Hospital, Finland Martin Walter,

Otto-von-Guericke-Universität

Magdeburg, Germany

*Correspondence:

Bharat B. Biswal, Department of Biomedical Engineering, New Jersey Institute of Technology, 607 Fenster hall, University Heights, Newark, NJ 07102, USA.

e-mail: bbiswal@yahoo.com

\begin{abstract}
Studies of brain functional connectivity have provided a better understanding of organization and integration of large-scale brain networks. Functional connectivity using restingstate functional magnetic resonance imaging ( $\mathrm{fMRI}$ ) is typically based upon the correlations of the low-frequency fluctuation of fMRI signals. Reproducible spatial maps in the brain have also been observed using the amplitude of low-frequency fluctuations (ALFF) in resting-state. However, little is known about the influence of the ALFF on the functional connectivity measures. In the present study, we analyzed resting-state $\mathrm{fMRI}$ data on 79 healthy old individuals. Spatial independent component analysis and regions of interest (ROIs) based connectivity analysis were performed to obtain measures of functional connectivity. ALFF maps were also calculated. First, voxel-matched inter-subject correlations were computed between back-reconstructed IC and ALFF maps. For all the resting-state networks, there was a consistent correlation between ALFF variability and network strengths (within regions that had high IC strengths). Next, inter-subject variance of correlations across 160 functionally defined ROls were correlated with the corresponding ALFF variance. The connectivity of several ROIs to other regions were more likely to correlate with its own regional ALFF. These regions were mainly located in the anterior cingulate cortex, medial prefrontal cortex, precuneus, insula, basal ganglia, and thalamus. These associations may suggest a functional significance of functional connectivity modulations. Alternatively, the fluctuation amplitudes may arise from physiological noises, and therefore, need to be controlled when studying resting-state functional connectivity.
\end{abstract}

Keywords: ALFF, basal ganglia, brain network, default mode network, independent component analysis, insula, thalamus

\section{INTRODUCTION}

Studies of brain networks and functional connectivity have provided a better understanding of organization and integration of large-scale brain networks. After the initial observation that the motor cortex exhibits highly synchronized intrinsic fluctuations during the absence of specific tasks (Biswal et al., 1995), the restingstate functional connectivity has emerged as a promising approach to investigate the functional integration of the brain. Studies using seed-based correlations have shown that the resting-state BOLD signal of functionally related regions generally demonstrate high correlation coefficients (e.g., Cordes et al., 2000). Seed-based correlation analysis has since been used to define brain networks such as the default mode network (DMN; Greicius et al., 2003), and to study the functional parcellation of specific brain structures, such as the cingulate cortex (Margulies et al., 2007), basal ganglia (Di Martino et al., 2008), and insula (Taylor et al., 2009).

As an alternative to seed-based analysis, where the region of interest is known, researchers have used independent component analysis (ICA), a data driven methodology to decompose the brain into spatially independent networks (McKeown et al., 1998). ICA simultaneously investigate multiple networks such as the DMN, salience, left/right executive, attention, motor, and visual networks (Greicius et al., 2004; Beckmann et al., 2005) and several successful applications have been reported in mental diseases (e.g., Greicius et al., 2004; Veer et al., 2010; Westlye et al., 2011).

The studies of functional connectivity and networks generally rely on the correlations and relative independence of lowfrequency fluctuation signals of resting-state functional magnetic resonance imaging (fMRI). However, the influences of restingstate fMRI signal fluctuation amplitude on the measures of functional connectivity and networks have largely been ignored. Theoretically, the correlation coefficient should be independent of the scale of the signals. However, the reliability of fMRI signals might be associated with the level of noises as well as meaningful neuronal functions (e.g., Sirotin and Das, 2009). Therefore, the fluctuation amplitudes may indeed affect the functional connectivity and network measures.

The "noise" of the brain has been shown to characterize the developing (McIntosh et al., 2008) and the aging (Garrett et al., 2010,2011 ) brain, and the variability of the noise has been shown to explain behavioral variability (for a review, see McIntosh et al., 2010). On the other hand, the resting-state fMRI is susceptible to many sources of noise such as head motion (Power et al., 2012; Van Dijk et al., 2012), respiration, and heartbeat (Birn et al., 2006, 2008; Chang et al., 2008). Data processing strategies were found to significantly affect connectivity measures (Weissenbacher 
et al., 2009; Saad et al., 2012), which implies that the connectivity results are still largely influenced by different sources of noise even after following careful processing procedures. Taken together, a better understanding of how the resting-state fMRI fluctuation amplitude affect functional connectivity and networks is warranted.

The fluctuations of resting-state BOLD signals are generally observed to be present between 0.01 and $0.08 \mathrm{~Hz}$ frequency band (Biswal et al., 1995). The amplitude of resting-state BOLD fluctuations is usually calculated in this low-frequency band, which has been termed as the amplitude of low-frequency fluctuations (ALFF, Zang et al., 2007). Higher ALFF in resting-state have been shown in regions constituting the DMN (Zang et al., 2007), suggesting that ALFF to some extent reflects neural activity. In addition, recent studies have observed an overlap between changes in regional ALFF and functional connectivity in several brain regions in stuttering (Xuan et al., 2012) and seasonal affective disorder subjects (Abou Elseoud et al., 2012). These studies suggest a relationship between ALFF and functional connectivity; however, the extent and selectivity of this association has not been investigated.

In the present study, we aimed to systematically examine the relationships between ALFF and resting-state connectivity. A large dataset of healthy old subjects were analyzed so that the intersubject variability of ALFF and connectivity was maximized. First, spatial ICA was performed on the resting-state fMRI data to identify resting-state networks. These networks were correlated with regional ALFFs in a voxel-wise manner to examine whether the inter-subject variability of the network strengths were correlated with ALFFs. Second, functional connectivity across 160 regions of interest (ROIs) were calculated. The functional connectivity was correlated with ALFF to examine whether the local amplitude fluctuations affect the strength of connectivity. We hypothesize that the strength of connectivity of ICA and ROI based analyses would be correlated to the local ALFF. In addition, the correlations were examined across different networks and connectivity pairs to determine whether these associations were across the entire brain or specific to selective networks.

\section{MATERIALS AND METHODS RESTING-STATE MRI DATA}

Resting-state fMRI and anatomical MRI data were obtained on a sample of old male subjects. After removing data with large head motion, 79 subjects were included with a mean age of 80.3 years (range from 65 to 92) for further analysis. A 3.0-T Siemens Magnetom Tim Trio scanner equipped with a 12-channel head coil (Erlangen, Germany) was used to acquire the MR images. All the functional and anatomical images were scanned parallel to the anterior commissureposterior commissure line. The resting-state data were scanned for $500 \mathrm{~s}$ with a TR of $2.5 \mathrm{~s}$, resulting in 200 images for each subject. The scanning parameters were as follows: $\mathrm{TE}=27$; acquisition matrix $=64 \times 64$; flip angle $=77^{\circ}$; slices $=43$; spatial resolution $=3.44 \mathrm{~mm} \times 3.44 \mathrm{~mm} \times 3.40 \mathrm{~mm}$. High resolution MPRAGE anatomical images were also acquired with the scanning parameters as follows: $\mathrm{TR}=2530 \mathrm{~ms}$; $\mathrm{TE}=3.5 \mathrm{~ms}$; flip angle $=7^{\circ}$; resolution $=1 \mathrm{~mm} \times 1 \mathrm{~mm} \times 1 \mathrm{~mm}$ (no gap).

\section{DATA ANALYSIS}

\section{Preprocessing}

The functional and anatomical image preprocessing were performed using SPM8 toolbox ${ }^{1}$ under MATLAB7.7 software $^{2}$. The first two functional images were discarded. Then, the remaining functional images were motion corrected and coregistered to the subjects' own anatomical images. The anatomical images were segmented using the new segmentation routine in SPM8. The deformation field maps obtained in segmentation were used to normalize all the functional images into standard Montreal Neurological Institute (MNI) space. For each voxel, the six rigid body head motion parameters, the first five eigenvectors from white matter (WM) signals, and the first five eigenvectors from cerebrospinal fluid (CSF) signals were regressed out using linear regression. The WM and CSF masks were defined for each subject using the segmented WM and CSF images thresholded at $p>0.99$. Finally, all the functional images were spatially smoothed using a Gaussian kernel of $8 \mathrm{~mm}$ full width at half maximum (FWHM).

\section{Calculation of ALFF}

Amplitude of low-frequency fluctuations maps were calculated between 0.01 and $0.08 \mathrm{~Hz}$ band using Resting-State fMRI Data Analysis Toolkit V1.6 (REST; Song et al., 2011). The ALFF maps were then divided by whole brain mean ALFF values to normalize the global effects.

\section{Relationships between network strength and ALFF}

Spatial ICA was conducted to define intrinsic networks using the Group ICA of fMRI Toolbox (GIFT) ${ }^{3}$ (Calhoun et al., 2001). Twenty components were extracted. Resting-state networks were visually identified according to the literature (Biswal et al., 2010; Cole et al., 2010). These ICs were back-reconstructed to each subject using group ICA algorithm, resulting in 20 IC maps for each subject (Erhardt et al., 2011). To examine whether there was a consistent network effect across subjects, voxel-wise one-sample $t$ tests was performed for each of the networks. The resulting $t$ maps were thresholded at $|t|>3.42(p<0.001)$.

A voxel-matched correlation analysis was used to study the relationships between resting-state network strengths and ALFFs (similar to Mennes et al., 2010, 2011). For each voxel, network strengths of an IC were correlated with ALFFs across all subjects using Pearson's correlation coefficient. The correlation maps were calculated separately for each of the network maps. Some voxels within an IC had negative value which reflects a negative relationship between a given voxel to the corresponding IC. Therefore, negative correlation between ALFF and negative IC strength is equivalent to positive correlation between ALFF and positive IC strength.

The resulting $r$ maps were thresholded at $|r|>0.364$ $(p<0.001)$. Because the aim of the current analysis was to show the overall correlation patterns, we did not use multiple comparison correction. However, a Monte Carlo simulation using AlphaSim ${ }^{4}$ indicated that a cluster exceeding 24 voxels were

\footnotetext{
${ }^{1}$ http://www.fil.ion.ucl.ac.uk/spm/

${ }^{2}$ http://www.mathworks.com/

${ }^{3}$ http://icatb.sourceforge.net/

${ }^{4}$ http://afni.nimh.nih.gov/pub/dist/doc/manual/AlphaSim.pdf
} 
significant at $p<0.05$ after a whole brain multiple comparison correction. This analysis shows that most of our large clusters reported in the results were still significant even after multiple comparison correction.

\section{Relationships between functional connectivity and ALFF}

Mean time series from 160 functionally defined ROIs were calculated within spherical ROIs with $8 \mathrm{~mm}$ radius (Dosenbach et al., 2010). These 160 ROIs were also assigned into six networks according to a modularity analysis of resting-state data (Dosenbach et al., 2010), including the cerebellar, cingulo-opercular, DMN, frontoparietal, occipital, and sensorimotor networks (see Table A1 in Appendix for details). Then, functional connectivity matrices were calculated for each subject using Pearson's correlation coefficient across 160 ROIs. The connectivity matrices were transformed into Fisher's $z$. For each of the ROI, the Fisher's $z$ scores between a given ROI to other ROIs were correlated with ALFF value of the given ROI.
To identify which ROI's local ALFF were more likely to correlate with connectivity, the correlations were thresholded at $|r|>0.364$ $(p<0.001)$. Then, we selected the ROIs with local ALFFs that were correlated with more than 30 significant connectivity between the given ROI and other ROIs. These ROIs and the corresponding connections with other ROIs were visualized using BrainNet Viewer ${ }^{5}$.

\section{RESULTS}

\section{RELATIONSHIPS BETWEEN NETWORK STRENGTH AND ALFF}

Out of the 20 ICs, 8 ICs were identified which corresponded to the 8 networks described by Cole et al. (2010), including the DMN, left and right executive, attention, salience, motor, visual, and fronto-parietal opercular networks (the left column of Figure 1). The voxel-matched correlations between network strengths and ALFFs for the eight networks were shown in the right column.

${ }^{5}$ http://www.nitrc.org/projects/bnv/
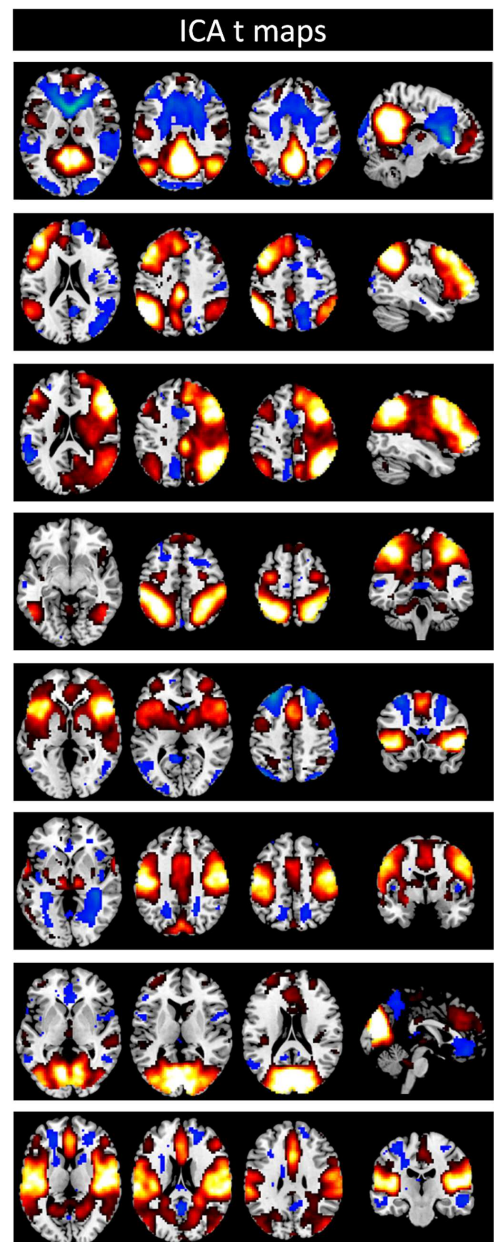

Negative

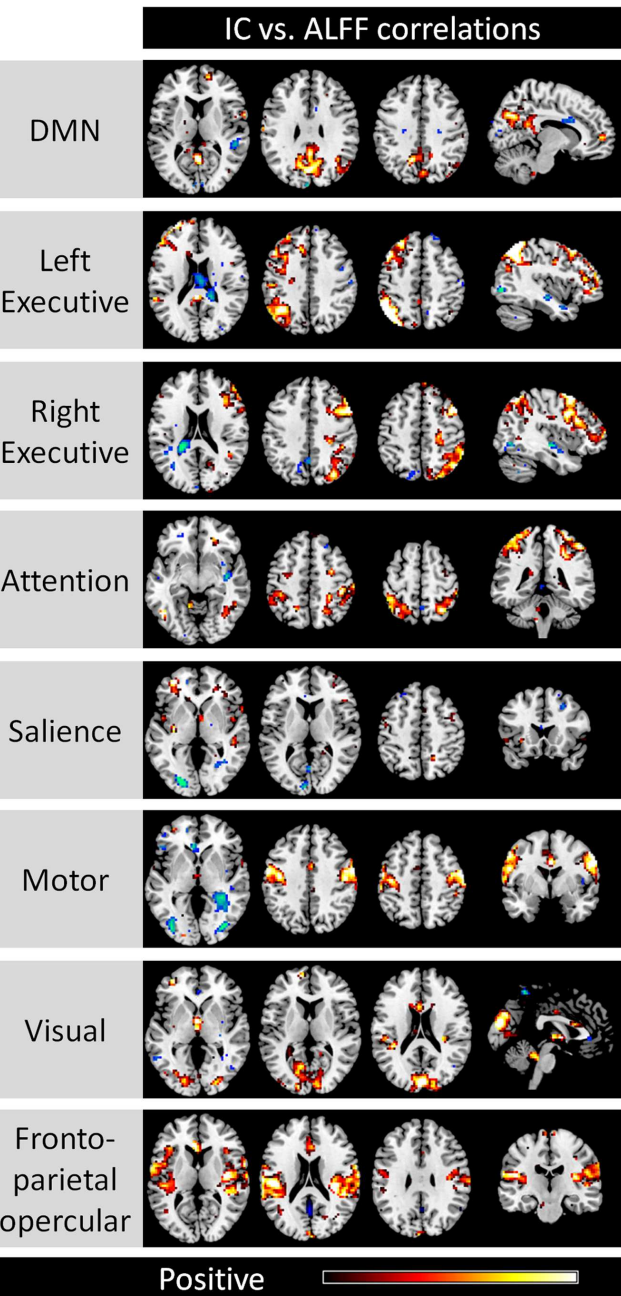

displayed range is absolute $t$ value between 3.42 and 20 , and for the correlation maps, display range is absolute $r$ value between 0.364 and 0.6. Hot and cold colors encode positive and negative effects, respectively. 
Strong correlations between network strengths and ALFFs were generally observed within each network with less spatial extent when using the compatible statistical threshold of $p<0.001$. For the DMN network, correlations between network strengths and ALFFs were observed in the posterior cingulate cortex/precuneus, medial prefrontal cortex (MPFC), and the right inferior parietal lobule/superior temporal gyrus. Within the left and right executive network, correlations were identified in the left and right dorsolateral prefrontal cortex and superior parietal lobule, respectively. The attention network demonstrated correlations within the bilateral superior parietal lobule and middle temporal gyrus. In the salience network, although clusters of high correlations were unapparent, there were small clusters within the bilateral insula and inferior frontal gyrus. For the motor network, correlations were observed in the bilateral sensorimotor cortex and supplementary motor area. In the visual network, correlations were identified primarily in the visual cortex. Lastly, correlations were observed in the bilateral insula/inferior frontal gyrus, and cingulate cortex within the fronto-parietal opercular network.

In addition to the eight ICs, four other ICs were considered to be meaningful brain networks (the left column of Figure 2). IC 12 was mainly comprised of the bilateral insula, bilateral anterior temporal lobe, bilateral hippocampal gyrus, and bilateral amygdala. IC 14 included regions within the bilateral superior frontal gyrus, medial frontal gyrus, and bilateral inferior parietal lobe, whereas IC 15 was mostly within the bilateral temporal lobe. IC 18 was mainly located in the MPFC, anterior cingulate cortex, and posterior cingulate cortex. High correlation between IC strengths and regional ALFFs were also observed in these regions of each network, respectively (right column).

We classified the remaining eight ICs as components related to noise. Voxels with high values within these ICs were mainly located in the CSF, WM, or large vessels (see Figure A1 in Appendix). We also observed high correlations between these IC strengths and ALFFs.

\section{RELATIONSHIPS BETWEEN FUNCTIONAL CONNECTIVITY AND ALFF}

The mean connectivity matrix across 160 ROIs is illustrated in the left panel of Figure 3. Even with strong connectivity coefficient values, we observed higher connectivity within each network compared with between networks. These ROIs were sorted by their six network affiliations (see Table A1 in Appendix), and high correlation values within the networks are evident as subsquares along the mean connectivity matrix diagonal, for example the cerebellar network (ROI 1-18), DMN (ROI 51-84), fronto-parietal network (ROI 85-105), occipital network (ROI 106-127), and sensorimotor network (ROI 128-160). However, we did not observe strong within network connectivity of the cingulo-opercular network (ROI 19-50).

The correlation between the ALFF of a given ROI and the connectivity between the given ROI with other ROIs are illustrated in the middle panel of Figure 3. The matrix was thresholded $(|r|>0.364$, i.e., $p<0.001)$ to determine which correlation between the ALFF of a given ROI and its connectivity were statistically significant. The right panel of Figure 3 demonstrates that the matrix was asymmetrical with respect to the diagonal which suggests that ALFFs of both ROIs within a pair affect functional connectivity differently. It also demonstrates that ALFF of specific ROIs were more likely to influence the connectivity between these specific ROIs with other ROIs.

The number of positive and negative correlations correlated with the local ALFF was tabulated (Figure 4) to identify the regions where the local ALFF were more likely to affect connectivity. We set an arbitrary threshold of $n>30$ to identify these regions (see

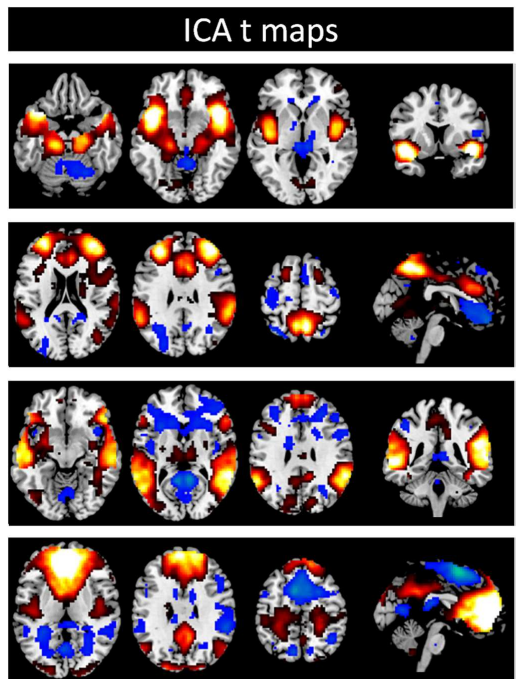

Negative
IC 12

IC vs. ALFF correlations

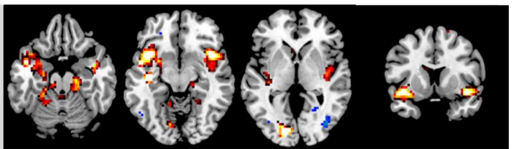

IC 14

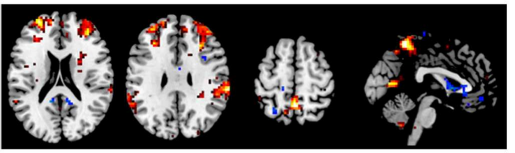

IC 15

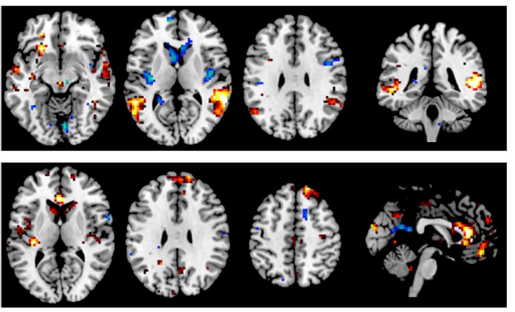

Positive
FIGURE 2 | Other four networks identified by spatial ICA (left column) and voxel-wise correlations between network strengths and ALFFs (right column). All maps are thresholded at $p<0.001$. For the ICA $t$ maps, display range is absolute $t$ value between 3.42 and 20 , and for the correlation maps, display range is absolute $r$ value between 0.364 and 0.6 . Hot and cold colors encode positive and negative effects, respectively. 


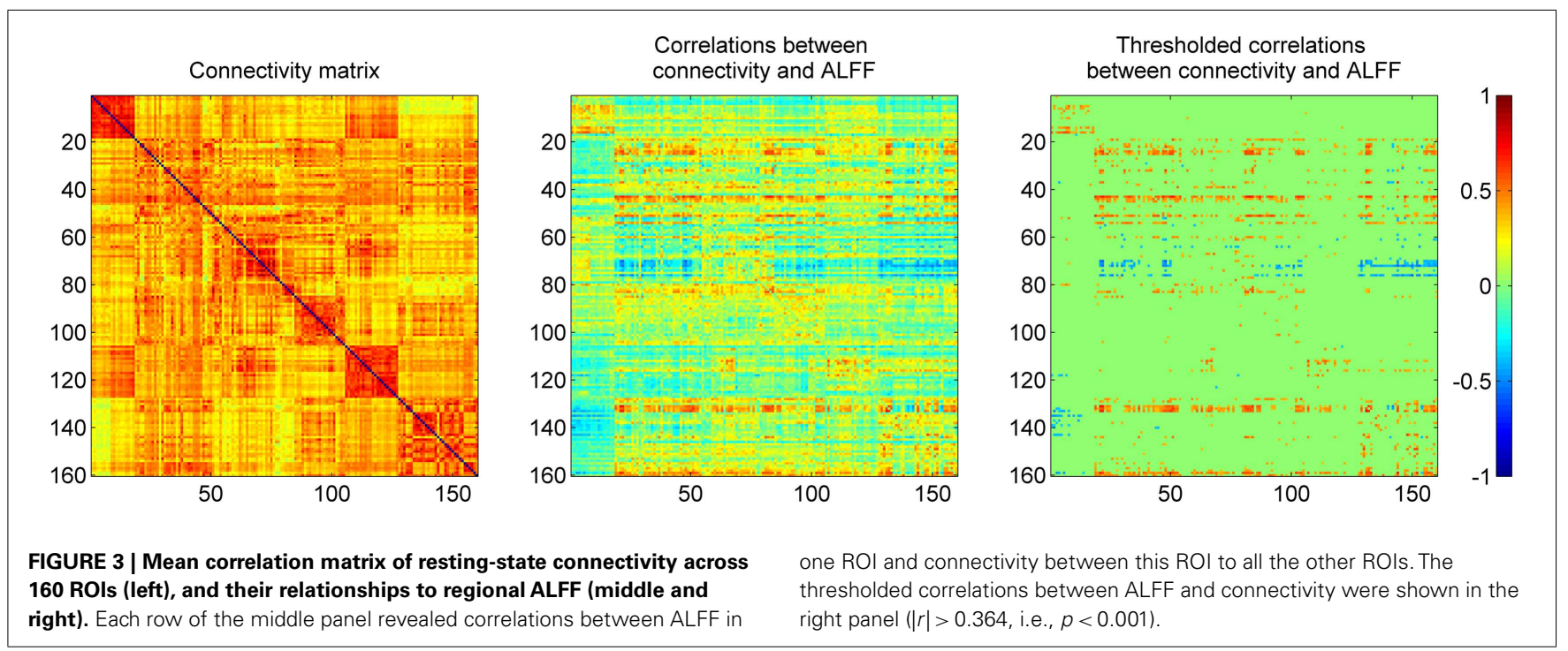

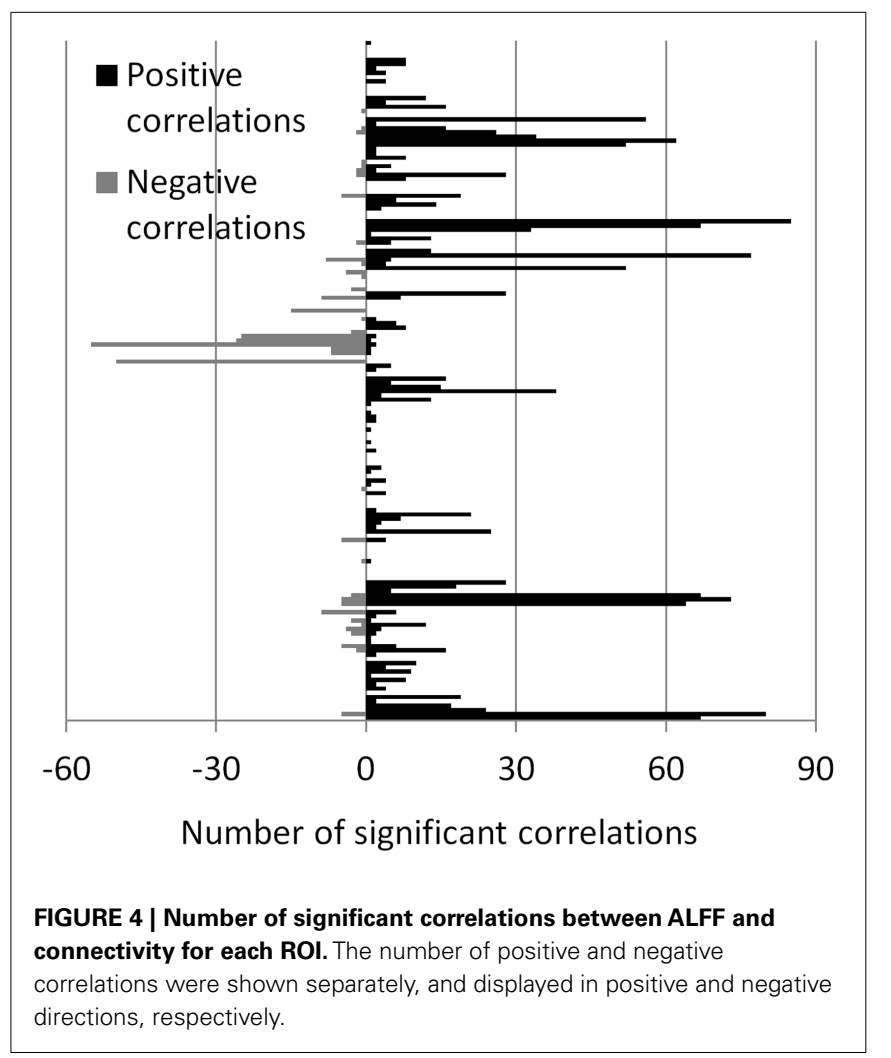

Table 1). Fifteen ROIs revealed more than 30 connections that were positively correlated with ALFFs from the corresponding ROIs, while two ROIs revealed more than 30 connections that were negatively correlated with ALFFs from the corresponding ROIs.

These ROIs were categorized into four groups based on their spatial approximations and affiliated networks. The first group of ROIs were located in the MPFC and anterior cingulate cortex (ACC) (Figure 5A). These five ROIs were either a part of the DMN or the cingulo-opercular network as described by
Table 1 | ROls that have more than $\mathbf{3 0}$ connections that are correlated with the corresponding regional ALFF.

\begin{tabular}{lclrrr}
\hline Label & ROI \# & Network & \multicolumn{2}{c}{ MNI coordinates } \\
\cline { 4 - 6 } & & & $\boldsymbol{x}$ & $\boldsymbol{y}$ & $\mathbf{z}$ \\
\hline POSITIVE EFFECTS & & & & \\
ACC & 19 & Cingulo-opercular & -2 & 30 & 27 \\
aPFC & 23 & Cingulo-opercular & 27 & 49 & 26 \\
Basal ganglia & 24 & Cingulo-opercular & 14 & 6 & 7 \\
Basal ganglia & 25 & Cingulo-opercular & -20 & 6 & 7 \\
Thalamus & 43 & Cingulo-opercular & -12 & -3 & 13 \\
Thalamus & 44 & Cingulo-opercular & -12 & -12 & 6 \\
Thalamus & 45 & Cingulo-opercular & 11 & -12 & 6 \\
ACC & 51 & Default & 9 & 39 & 20 \\
aPFC & 54 & Default & -25 & 51 & 27 \\
vmPFC & 83 & Default & -11 & 45 & 17 \\
Mid insula & 131 & Sensorimotor & -42 & -3 & 11 \\
Mid insula & 132 & Sensorimotor & -36 & -12 & 15 \\
Mid insula & 133 & Sensorimotor & 33 & -12 & 16 \\
vFC & 159 & Sensorimotor & 43 & 1 & 12 \\
vFC & 160 & Sensorimotor & -55 & 7 & 23 \\
NEGATIVE EFFECTS & & & & \\
Precuneus & 72 & Default & 5 & -50 & 33 \\
Precuneus & 76 & Default & -6 & -56 & 29 \\
& & & & &
\end{tabular}

Dosenbach et al. (2010); however, these nearby ROIs exhibited similar correlation patterns. The connectivity between the five ROIs with other DMN, fronto-parietal, cingulo-opercular, sensorimotor, and occipital regions demonstrated positive correlations with local ALFF (Figures 5E,I). The second set of ROIs were located in the precuneus (Figure $5 \mathbf{B}$ ), and the connectivity of these ROIs to cingulo-opercular, fronto-parietal, and sensorimotor regions were negatively correlated with local ALFF (Figures 5F,J). The third set was comprised of five ROIs in the bilateral putamen, caudate, and thalamus (Figure 5C), and their 


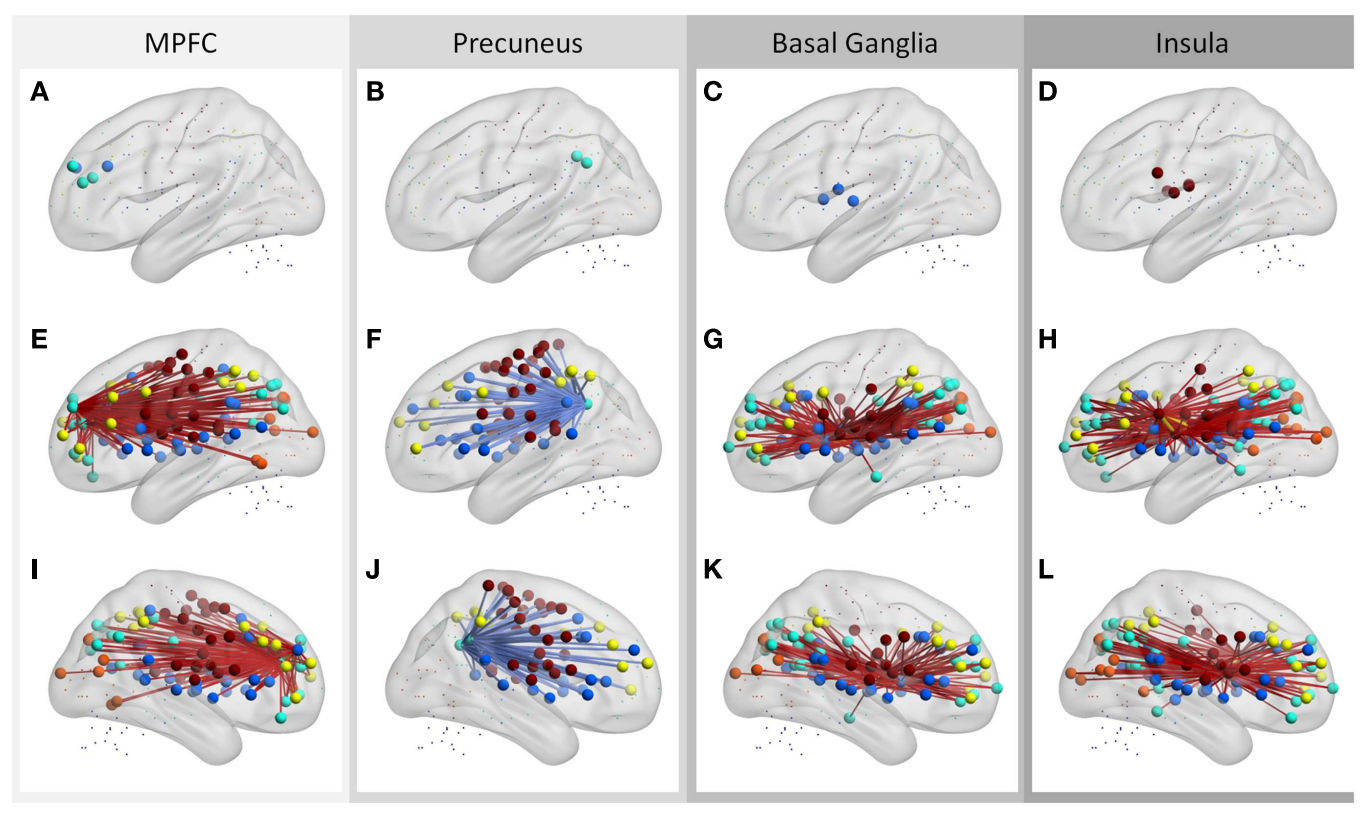

FIGURE 5 | Regions that have more than 30 connections correlated with regional ALFF (A-D), and their associated connections (E-L). The ROls are stratified into four sets according to their affiliated networks and connectivity behavior (one for each column). Middle and bottom rows showed left and right lateral views, respectively. Hot and cold colors of connections indicate positive and negative correlations. Color codes of the ROls: blue, cingulo-opercular network; cyan, DMN; yellow, fronto-parietal network; orange, occipital network; brown, sensorimotor network. connectivity to the DMN, fronto-parietal, cingulo-opercular, and occipital regions revealed positive correlations with local ALFF (Figures 5G,K). The fourth group of ROIs were located at the bilateral insula and ventral fontal regions (Figure 5D), and their connectivity to the DMN, fronto-parietal, cingulo-opercular, and occipital regions revealed positive correlations with local ALFF (Figures 5H,L).

\section{DISCUSSION}

The current analysis demonstrates that the network strengths as measured by ICA were selectively correlated with ALFFs within the corresponding network. The network strength measured by ICA reflects the extent that a particular voxel correlates with the whole IC. Thus, the correlations between ALFFs and network strengths imply that the within network connectivity are correlated with the local fluctuation amplitudes. The relationship between ICA and ALFF were replicated by directly correlating ALFFs with connectivity measured via correlations. Within each network, ALFFs were positively correlated with the connectivity and were demonstrated as squares within each network nearby the diagonal of the matrix (see the right panel of Figure 3, e.g., cerebellar and sensorimotor networks). Interestingly, the correlation between ALFFs and connectivity were not restricted to within network but extends to between network connectivity. The functional connectivity of regions, particularly the MPFC, ACC, precuneus, basal ganglia, thalamus, and insula, with other regions were widely spread in the whole brain and suggest a special role of these regions in functional connectivity pattern.

The association between local fluctuations and connectivity may simply reflect that the BOLD signals are more reliable with less noise. However, given that the correlations are not uniform across the whole brain and that selective correlations are between specific regional ALFFs and connectivity, these associations may suggest functional significance. One possible explanation is that these selective regions may be involved in transmitting information to various brain regions, such that the greater the neural activity results in larger regional amplitude of fluctuations, and greater connectivity between these regions to other regions. In addition, the variances of ALFF may reflect different levels of neurotransmitters that give rise to functional connectivity variances. The later notion can be tested by combining resting-state fMRI with magnetic resonance spectroscopy (MRS) or positron emission tomography (PET) (Horn et al., 2010; Hahn et al., 2011; Cole et al., 2012; Kapogiannis et al., 2013).

Alternatively, it is also possible that these regions are more likely to be impacted by physiological noise. Even though ALFF is considered to be a measure of amplitude of neural activity, our recent studies have shown that ALFF is highly correlated with neurovascular response of breath holding task (Biswal et al., 2007; Di et al., 2013). In addition, the regions that demonstrate high correlations between ALFF and connectivity were also the regions that were more likely to be affected by physiological noise due to the adjacent large vessels, including the MPFC and precuneus, and insula (Di et al., 2013). These physiological noises may also influence functional connectivity (Birn et al., 2006, 2008; Chang et al., 2008), and therefore, reflect the common sources of physiological noise that affects both measures. Consistent with this notion, the ICs that reflected physiological noises exhibited high correlations in the regions located in the CSF, WM, and large vessels (see Figure A1 in Appendix). However, for the ICs that reflect meaningful neural 
networks, the correlations between ALFF and network strength may reflect both neural and noise contributions.

The first two sets of ROIs exhibiting correlations between connectivity and ALFF were within the DMN, including the MPFC/ACC regions, and precuneus. These regions are also defined as the structural core of the human brain that has the most anatomical connections to other brain regions (Hagmann et al., 2008). Most interestingly, the correlations between regional ALFF and connectivity showed reversed relationships between the prefrontal regions and precuneus. The connectivity between the two ROIs in precuneus with other regions was negatively correlated with the precuneus ALFF. These brain regions were task positive networks such as the sensorimotor, fronto-parietal, and cinguloopercular networks. The connectivity between DMN and task positive networks is generally negative (Fox et al., 2005), which suggests that greater regional ALFF is associated with greater negative connectivity between DMN and task positive networks. In addition, we did not apply global scaling on the current dataset in order to prevent artificial negative correlations and no negative connectivity was observed. Thus, the negative relationship between connectivity (DMN and task positive networks) and ALFF is not due to preprocessing of the data. In contrast, the connectivity between MPFC/ACC ROIs and other regions revealed positive correlations with ALFF. MPFC/ACC ROIs were correlated with regions within other areas of the DMN, and with regions of the fronto-parietal, sensorimotor, cingulo-opercular, and occipital networks. These different correlation pattern suggests that the modulation of connectivity may involve different underlying mechanisms, e.g., via excitatory and inhibitory neurotransmitter modulations. Glutamate concentration, which reflects excitatory mechanisms in the ACC (Horn et al., 2010) and posteromedial cortex (Kapogiannis et al., 2013) has been shown to positively modulate the restingstate functional connectivity. In contrast, GABA concentration, which reflects inhibitory mechanisms, in the posteromedial cortex has been shown to negatively correlate with the resting-state functional connectivity. However, the links between the amplitude of fluctuations and neurotransmitter concentrations is still largely unknown, thus require further studies.

The other two sets of ROIs include the basal ganglia, thalamus, insula, and adjacent sensorimotor regions. Previous studies have demonstrated a widely spread functional connectivity of these regions to other brain regions (e.g., Di Martino et al., 2008; Taylor

\section{REFERENCES}

Abou Elseoud, A., Nissilä, J., Liettu, A., Remes, J., Jokelainen, J., Takala, T., et al. (2012). Altered restingstate activity in seasonal affective disorder. Hum. Brain Mapp. doi:10.1002/hbm.22164

Beckmann, C. F., DeLuca, M., Devlin, J. T., and Smith, S. M. (2005). Investigations into resting-state connectivity using independent component analysis. Philos. Trans. R. Soc. Lond. B Biol. Sci. 360, 1001-1013.

Birn, R. M., Diamond, J. B., Smith, M. A., and Bandettini, P. A.

et al., 2009; Cauda et al., 2011; Tang et al., 2011). The positive correlations between connectivity and ALFF suggest a special role of these regions in functional connectivity pattern.

A practical implication of the present result is that when studying resting-state functional connectivity or networks, ALFF may be a potential confounding variable that needs to be taken into account. ALFF has been widely used to study the "baseline" activity of a wide spectrum of psychological states and mental diseases, for example aging (Biswal et al., 2010; Yan et al., 2011), schizophrenia (Hoptman et al., 2010; Huang et al., 2010), and attention deficit hyperactivity disorder (ADHD; Zang et al., 2007). Distributed differences of ALFF have been observed to be associated with different pathologies and mental states. On the other hand, increasingly studies have been conducted to investigate brain functional connectivity alterations in mental diseases using both seed-based correlation and spatial ICA (e.g., Greicius et al., 2004; Castellanos et al., 2008; Veer et al., 2010; Westlye et al., 2011). Although these differences are presumed to reflect the group differences in resting-state connectivity and networks, ALFF was not controlled by previous studies. Therefore, the underlying group differences in functional connectivity that have been reported by previous studies may be due to the unrestrained ALFF. By including ALFF as covariance, Abou Elseoud et al. (2012) demonstrated increased connectivity, but less number of voxels in the visual network. Thus, the current result and that of Abou Elseoud et al. (2012) raises a concern regarding ALFF as a potential confound when study functional connectivity and network. More specifically, our data suggests that one should be cautious when interpreting seed-based correlations of regions that are more likely to be affected by ALFF, such as the precuneus, MPFC, basal ganglia, thalamus, and insula.

The present study only analyzed a sample of old individuals because old subjects typically demonstrate larger variance of functional connectivity and the associations of functional connectivity with ALFF may be easier to identify. Even though we believe that the current results will also hold for younger individuals, further studies investigating younger individuals is needed to determine whether the relationship between local fluctuation amplitudes and functional connectivity generalizes to young population.

\section{ACKNOWLEDGMENTS}

This study was supported by a National Institute of Health grant 5R01AG032088.

echo-planar MRI. Magn. Reson. Med. 34, 537-541.

Biswal, B. B., Kannurpatti, S. S., and Rypma, B. (2007). Hemodynamic scaling of fMRI-BOLD signal: validation of low-frequency spectral amplitude as a scalability factor. Magn. Reson. Imaging 25, 1358-1369.

Biswal, B. B., Mennes, M., Zuo, X. N., Gohel, S., Kelly, C., Smith, S. M., et al. (2010). Toward discovery science of human brain function. Proc. Natl. Acad. Sci. U.S.A. 107, 4734-4739.

Calhoun, V. D., Adali, T., Pearlson, G. D., and Pekar, J. J. (2001). A method for making group inferences from functional MRI data using independent component analysis. Hum. Brain Mapp. 14, 140-151.

Castellanos, F. X., Margulies, D. S., Kelly, C., Uddin, L. Q., Ghaffari, M., Kirsch, A., et al. (2008). Cingulateprecuneus interactions: a new locus of dysfunction in adult attentiondeficit/hyperactivity disorder. Biol Psychiatry. 63, 332-337.

Cauda, F., D'Agata, F., Sacco, K., Duca, S., Geminiani, G., and Vercelli, A. (2011). Functional connectivity of the insula in the resting brain. $\mathrm{Neu}$ roimage $55,8-23$. 
Chang, C., Thomason, M. E., and Glover, G. H. (2008). Mapping and correction of vascular hemodynamic latency in the BOLD signal. Neuroimage 43, 90-102.

Cole, D. M., Beckmann, C. F., Searle, G. E., Plisson, C., Tziortzi, A. C., Nichols, T. E., et al. (2012). Orbitofrontal connectivity with resting-state networks is associated with midbrain dopamine D3 receptor availability. Cereb. Cortex 22, 2784-2793.

Cole, D. M., Smith, S. M., and Beckmann, C. F. (2010). Advances and pitfalls in the analysis and interpretation of resting-state FMRI data. Front. Syst. Neurosci. 4:8. doi:10.3389/fnsys.2010.00008

Cordes, D., Haughton, V. M., Arfanakis, K., Wendt, G. J., Turski, P. A., Moritz, C. H., et al. (2000). Mapping functionally related regions of brain with functional connectivity MR imaging. AJNR Am. J. Neuroradiol. 21, 1636-1644.

Di, X., Kannurpatti, S. S., Rypma, B., and Biswal, B. B. (2013). Calibrating BOLD fMRI activations with neurovascular and anatomical constraints. Cereb. Cortex 23, 255-263.

Di Martino, A., Scheres, A., Margulies, D. S., Kelly, A. M., Uddin, L. Q., Shehzad, Z., et al. (2008). Functional connectivity of human striatum: a resting state fMRI study. Cereb. Cortex 18, 2735-2747.

Dosenbach, N. U., Nardos, B., Cohen, A. L., Fair, D. A., Power, J. D., Church, J. A., et al. (2010). Prediction of individual brain maturity using fMRI. Science 329, 1358-1361.

Erhardt, E. B., Rachakonda, S., Bedrick, E. J., Allen, E. A., Adali, T., and Calhoun, V. D. (2011). Comparison of multi-subject ICA methods for analysis of fMRI data. Hum. Brain Mapp. 32, 2075-2095.

Fox, M. D., Snyder, A. Z., Vincent, J. L., Corbetta, M., Van Essen, D. C., and Raichle, M. E. (2005). The human brain is intrinsically organized into dynamic, anticorrelated functional networks. Proc. Natl. Acad. Sci. USA. 102, 9673-9678.

Garrett, D. D., Kovacevic, N., McIntosh, A. R., and Grady, C. L. (2010). Blood oxygen level-dependent signal variability is more than just noise. J. Neurosci. 30, 4914-4921.

Garrett, D. D., Kovacevic, N., McIntosh, A. R., and Grady, C. L. (2011). The importance of being variable. $J$. Neurosci. 31, 4496-4503.

Greicius, M. D., Krasnow, B., Reiss, A. L., and Menon, V. (2003). Functional connectivity in the resting brain: a network analysis of the default mode hypothesis. Proc. Natl. Acad. Sci. U.S.A. 100, 253-258.

Greicius, M. D., Srivastava, G., Reiss, A. L., and Menon, V. (2004). Defaultmode network activity distinguishes Alzheimer's disease from healthy aging: evidence from functional MRI. Proc. Natl. Acad. Sci. U.S.A. 101, 4637-4642.

Hagmann, P., Cammoun, L., Gigandet, X., Meuli, R., Honey, C. J., Wedeen, V. J., et al. (2008). Mapping the structural core of human cerebral cortex. PLoS Biol. 6:e159. doi:10.1371/journal.pbio.0060159

Hahn, A., Wadsak, W., Windischberger, C., Baldinger, P., Hoflich, A. S., Losak, J., et al. (2011). Differential modulation of the default mode network via serotonin-1A receptors. Proc. Natl. Acad. Sci. U.S.A. 109, 2619-2624.

Hoptman, M. J., Zuo, X. N., Butler, P. D., Javitt, D. C., D’Angelo, D., Mauro, C. J., et al. (2010). Amplitude of low-frequency oscillations in schizophrenia: a resting state fMRI study. Schizophr. Res. 117, 13-20.

Horn, D. I., Yu, C., Steiner, J., Buchmann, J., Kaufmann, J., Osoba, A., et al. (2010). Glutamatergic and restingstate functional connectivity correlates of severity in major depression - the role of pregenual anterior cingulated cortex and anterior insula. Front. Syst. Neurosci. 4:33. doi: $10.3389 /$ fnsys. 2010.00033

Huang, X. Q., Lui, S., Deng, W., Chan, R. C., Wu, Q. Z., Jiang, L. J., et al. (2010). Localization of cerebral functional deficits in treatmentnaive, first-episode schizophrenia using resting-state fMRI. Neuroimage 49, 2901-2906.

Kapogiannis, D., Reiter, D. A., Willette, A. A., and Mattson, M. P. (2013). Posteromedial cortex glutamate and GABA predict intrinsic functional connectivity of the default mode network. Neuroimage 64, 112-119.

Margulies, D. S., Kelly, A. M., Uddin, L. Q., Biswal, B. B., Castellanos, F. X., and Milham, M. P. (2007). Mapping the functional connectivity of anterior cingulate cortex. Neuroimage 37, 579-588.

McIntosh, A. R., Kovacevic, N., and Itier, R. J. (2008). Increased brain signal variability accompanies lower behavioral variability in development. PLoS Comput. Biol. 4:e1000106. doi:10.1371/journal.pcbi.1000106

McIntosh, A. R., Kovacevic, N., Lippe, S., Garrett, D., Grady, C., and Jirsa, V. (2010). The development of a noisy brain. Arch. Ital. Biol. 148, 323-337.

McKeown, M. J., Jung, T. P., Makeig, S., Brown, G., Kindermann, S. S., Lee, T. W., et al. (1998). Spatially independent activity patterns in functional MRI data during the stroop colornaming task. Proc. Natl. Acad. Sci. U.S.A. 95, 803-810.

Mennes, M., Kelly, C., Zuo, X. N., Di Martino, A., Biswal, B. B., Castellanos, F. X., et al. (2010). Interindividual differences in restingstate functional connectivity predict task-induced BOLD activity. Neuroimage 50, 1690-1701.

Mennes, M., Zuo, X. N., Kelly, C., Di Martino, A., Zang, Y. F., Biswal, B., et al. (2011). Linking interindividual differences in neural activation and behavior to intrinsic brain dynamics. Neuroimage 54, 2950-2959.

Power, J. D., Barnes, K. A., Snyder, A. Z., Schlaggar, B. L., and Petersen, S. E. (2012). Spurious but systematic correlations in functional connectivity MRI networks arise from subject motion. Neuroimage 59, 2142-2154.

Saad, Z. S., Gotts, S. J., Murphy, K. Chen, G., Jo, H. J., Martin, A., et al. (2012). Trouble at rest: how correlation patterns and group differences become distorted after global signal regression. Brain Connect. 2, 25-32.

Sirotin, Y. B., and Das, A. (2009). Anticipatory haemodynamic signals in sensory cortex not predicted by local neuronal activity. Nature 457, 475-479.

Song, X. W., Dong, Z. Y., Long, X. Y., Li, S. F., Zuo, X. N., Zhu, C. Z., et al. (2011). REST: a toolkit for resting-state functional magnetic resonance imaging data processing. PLOS ONE 6:e25031. doi:10.1371/journal.pone.0025031

Tang, L., Ge, Y., Sodickson, D. K. Miles, L., Zhou, Y., Reaume, J., et al. (2011). Thalamic resting-state functional networks: disruption in patients with mild traumatic brain injury. Radiology 260, 831-840.

Taylor, K. S., Seminowicz, D. A., and Davis, K. D. (2009). Two systems of resting state connectivity between the insula and cingulate cortex. Hum. Brain Mapp. 30 2731-2745.

Van Dijk, K. R., Sabuncu, M. R., and Buckner, R. L. (2012). The influence of head motion on intrinsic functional connectivity MRI. Neuroimage 59, 431-438.

Veer, I. M., Beckmann, C. F., van Tol, M. J., Ferrarini, L., Milles, J., Veltman, D.
J., et al. (2010). Whole brain restingstate analysis reveals decreased functional connectivity in major depression. Front. Syst. Neurosci. 4:41. doi:10.3389/fnsys.2010.00041

Weissenbacher, A., Kasess, C., Gerstl, F., Lanzenberger, R., Moser, E., and Windischberger, C. (2009). Correlations and anticorrelations in resting-state functional connectivity MRI: a quantitative comparison of preprocessing strategies. Neuroimage 47, 1408-1416.

Westlye, E. T., Lundervold, A., Rootwelt, H., Lundervold, A. J., and Westlye, L. T. (2011). Increased hippocampal default mode synchronization during rest in middle-aged and elderly APOE $\varepsilon 4$ carriers: relationships with memory performance. J. Neurosci. 31, 7775-7783.

Xuan, Y., Meng, C., Yang, Y., Zhu, C., Wang, L., Yan, Q., et al. (2012). Resting-state brain activity in adult males who stutter. PLoS ONE 7:e30570. doi:10.1371/journal.pone.0030570

Yan, L., Zhuo, Y., Wang, B., and Wang, D. J. (2011). Loss of coherence of low frequency fluctuations of BOLD fMRI in visual cortex of healthy aged subjects. Open Neuroimag. J. 5, 105-111.

Zang, Y. F., He, Y., Zhu, C. Z., Cao, Q. J., Sui, M. Q., Liang, M., et al. (2007). Altered baseline brain activity in children with ADHD revealed by resting-state functional MRI. Brain Dev. 29, 83-91.

Conflict of Interest Statement: The authors declare that the research was conducted in the absence of any commercial or financial relationships that could be construed as a potential conflict of interest.

Received: 14 December 2012; accepted: 18 March 2013; published online: 02 April 2013.

Citation: Di X, Kim EH, Huang C-C, Tsai S-J, Lin C-P and Biswal BB (2013) The influence of the amplitude of low-frequency fluctuations on resting-state functional connectivity. Front. Hum. Neurosci. 7:118. doi: 10.3389/fnhum.2013.00118 Copyright (c) 2013 Di, Kim, Huang, Tsai, Lin and Biswal. This is an openaccess article distributed under the terms of the Creative Commons Attribution License, which permits use, distribution and reproduction in other forums, provided the original authors and source are credited and subject to any copyright notices concerning any third-party graphics etc. 
APPENDIX

Table A1 | One hundred sixty functionally defined ROls used in the current study.

\begin{tabular}{|c|c|c|c|c|c|}
\hline ROI \# & $x$ & $y$ & $z$ & Label & Network \\
\hline 1 & -34 & -67 & -29 & Inf cerebellum & Cerebellum \\
\hline 2 & 32 & -61 & -31 & Inf cerebellum & Cerebellum \\
\hline 3 & -25 & -60 & -34 & Inf cerebellum & Cerebellum \\
\hline 4 & -37 & -54 & -37 & Inf cerebellum & Cerebellum \\
\hline 5 & 18 & -81 & -33 & Inf cerebellum & Cerebellum \\
\hline 6 & -6 & -79 & -33 & Inf cerebellum & Cerebellum \\
\hline 7 & -21 & -79 & -33 & Inf cerebellum & Cerebellum \\
\hline 8 & 33 & -73 & -30 & Inf cerebellum & Cerebellum \\
\hline 9 & -24 & -54 & -21 & Lat cerebellum & Cerebellum \\
\hline 10 & 21 & -64 & -22 & Lat cerebellum & Cerebellum \\
\hline 11 & -28 & -44 & -25 & Lat cerebellum & Cerebellum \\
\hline 12 & -34 & -57 & -24 & Lat cerebellum & Cerebellum \\
\hline 13 & 14 & -75 & -21 & Med cerebellum & Cerebellum \\
\hline 14 & 1 & -66 & -24 & Med cerebellum & Cerebellum \\
\hline 15 & -6 & -60 & -15 & Med cerebellum & Cerebellum \\
\hline 16 & -16 & -64 & -21 & Med cerebellum & Cerebellum \\
\hline 17 & 5 & -75 & -11 & Med cerebellum & Cerebellum \\
\hline 18 & -11 & -72 & -14 & Med cerebellum & Cerebellum \\
\hline 19 & -2 & 30 & 27 & $\mathrm{ACC}$ & Cingulo-opercular \\
\hline 20 & -41 & -47 & 29 & Angular gyrus & Cingulo-opercular \\
\hline 21 & 38 & 21 & -1 & Ant insula & Cingulo-opercular \\
\hline 22 & -36 & 18 & 2 & Ant insula & Cingulo-opercular \\
\hline 23 & 27 & 49 & 26 & aPFC & Cingulo-opercular \\
\hline 24 & 14 & 6 & 7 & Basal ganglia & Cingulo-opercular \\
\hline 25 & -20 & 6 & 7 & Basal ganglia & Cingulo-opercular \\
\hline 26 & -6 & 17 & 34 & Basal ganglia & Cingulo-opercular \\
\hline 27 & 11 & -24 & 2 & Basal ganglia & Cingulo-opercular \\
\hline 28 & 9 & 20 & 34 & $\mathrm{dACC}$ & Cingulo-opercular \\
\hline 29 & 54 & -31 & -18 & Fusiform & Cingulo-opercular \\
\hline 30 & 0 & 15 & 45 & $\mathrm{mFC}$ & Cingulo-opercular \\
\hline 31 & 37 & -2 & -3 & Mid insula & Cingulo-opercular \\
\hline 32 & -30 & -14 & 1 & Mid insula & Cingulo-opercular \\
\hline 33 & 32 & -12 & 2 & Mid insula & Cingulo-opercular \\
\hline 34 & -55 & -44 & 30 & Parietal & Cingulo-opercular \\
\hline 35 & 58 & -41 & 20 & Parietal & Cingulo-opercular \\
\hline 36 & -4 & -31 & -4 & Post cingulate & Cingulo-opercular \\
\hline 37 & -30 & -28 & 9 & Post insula & Cingulo-opercular \\
\hline 38 & 8 & -40 & 50 & Precuneus & Cingulo-opercular \\
\hline 39 & 42 & -46 & 21 & Sup temporal & Cingulo-opercular \\
\hline 40 & 43 & -43 & 8 & Temporal & Cingulo-opercular \\
\hline 41 & -59 & -47 & 11 & Temporal & Cingulo-opercular \\
\hline 42 & 51 & -30 & 5 & Temporal & Cingulo-opercular \\
\hline 43 & -12 & -3 & 13 & Thalamus & Cingulo-opercular \\
\hline 44 & -12 & -12 & 6 & Thalamus & Cingulo-opercular \\
\hline 45 & 11 & -12 & 6 & Thalamus & Cingulo-opercular \\
\hline 46 & -52 & -63 & 15 & TPJ & Cingulo-opercular \\
\hline 47 & -46 & 10 & 14 & $\mathrm{vFC}$ & Cingulo-opercular \\
\hline 48 & -48 & 6 & 1 & $\mathrm{vFC}$ & Cingulo-opercular \\
\hline 49 & 51 & 23 & 8 & $\mathrm{vFC}$ & Cingulo-opercular \\
\hline 50 & 34 & 32 & 7 & VPFC & Cingulo-opercular \\
\hline
\end{tabular}

\begin{tabular}{|c|c|c|c|c|c|}
\hline ROI \# & $x$ & $y$ & $z$ & Label & Network \\
\hline 51 & 9 & 39 & 20 & $\mathrm{ACC}$ & Default \\
\hline 52 & -48 & -63 & 35 & Angular gyrus & Default \\
\hline 53 & 51 & -59 & 34 & Angular gyrus & Default \\
\hline 54 & -25 & 51 & 27 & aPFC & Default \\
\hline 55 & 28 & -37 & -15 & Fusiform & Default \\
\hline 56 & -59 & -25 & -15 & Inf temporal & Default \\
\hline 57 & -61 & -41 & -2 & Inf temporal & Default \\
\hline 58 & 52 & -15 & -13 & Inf temporal & Default \\
\hline 59 & -36 & -69 & 40 & IPS & Default \\
\hline 60 & 0 & 51 & 32 & mPFC & Default \\
\hline 61 & 45 & -72 & 29 & Occipital & Default \\
\hline 62 & -9 & -72 & 41 & Occipital & Default \\
\hline 63 & -42 & -76 & 26 & Occipital & Default \\
\hline 64 & -28 & -42 & -11 & Occipital & Default \\
\hline 65 & -2 & -75 & 32 & Occipital & Default \\
\hline 66 & 10 & -55 & 17 & Post cingulate & Default \\
\hline 67 & -11 & -58 & 17 & Post cingulate & Default \\
\hline 68 & -8 & -41 & 3 & Post cingulate & Default \\
\hline 69 & 1 & -26 & 31 & Post cingulate & Default \\
\hline 70 & -5 & -52 & 17 & Post cingulate & Default \\
\hline 71 & -5 & -43 & 25 & Post cingulate & Default \\
\hline 72 & 5 & -50 & 33 & Precuneus & Default \\
\hline 73 & 11 & -68 & 42 & Precuneus & Default \\
\hline 74 & 9 & -43 & 25 & Precuneus & Default \\
\hline 75 & -3 & -38 & 45 & Precuneus & Default \\
\hline 76 & -6 & -56 & 29 & Precuneus & Default \\
\hline 77 & 23 & 33 & 47 & Sup frontal & Default \\
\hline 78 & -16 & 29 & 54 & Sup frontal & Default \\
\hline 79 & 46 & 39 & -15 & VIPFC & Default \\
\hline 80 & 6 & 64 & 3 & vmPFC & Default \\
\hline 81 & -6 & 50 & -1 & vmPFC & Default \\
\hline 82 & 9 & 51 & 16 & vmPFC & Default \\
\hline 83 & -11 & 45 & 17 & vmPFC & Default \\
\hline 84 & 8 & 42 & -5 & VmPFC & Default \\
\hline 85 & -1 & 28 & 40 & ACC & Fronto-parietal \\
\hline 86 & 29 & 57 & 18 & aPFC & Fronto-parietal \\
\hline 87 & -29 & 57 & 10 & aPFC & Fronto-parietal \\
\hline 88 & -42 & 7 & 36 & $\mathrm{dFC}$ & Fronto-parietal \\
\hline 89 & 40 & 17 & 40 & $\mathrm{dFC}$ & Fronto-parietal \\
\hline 90 & 44 & 8 & 34 & $\mathrm{dFC}$ & Fronto-parietal \\
\hline 91 & 40 & 36 & 29 & dIPFC & Fronto-parietal \\
\hline 92 & 46 & 28 & 31 & dIPFC & Fronto-parietal \\
\hline 93 & -44 & 27 & 33 & dIPFC & Fronto-parietal \\
\hline 94 & -48 & -47 & 49 & IPL & Fronto-parietal \\
\hline 95 & -41 & -40 & 42 & IPL & Fronto-parietal \\
\hline 96 & -53 & -50 & 39 & IPL & Fronto-parietal \\
\hline 97 & 44 & -52 & 47 & IPL & Fronto-parietal \\
\hline 98 & 54 & -44 & 43 & IPL & Fronto-parietal \\
\hline 99 & -32 & -58 & 46 & IPS & Fronto-parietal \\
\hline 100 & 32 & -59 & 41 & IPS & Fronto-parietal \\
\hline
\end{tabular}


Table A1 | Continued

\begin{tabular}{|c|c|c|c|c|c|}
\hline ROI \# & $x$ & $y$ & $z$ & Label & Network \\
\hline 101 & -35 & -46 & 48 & Post parietal & Fronto-parietal \\
\hline 102 & 42 & 48 & -3 & Vent aPFC & Fronto-parietal \\
\hline 103 & -43 & 47 & 2 & Vent aPFC & Fronto-parietal \\
\hline 104 & 39 & 42 & 16 & vIPFC & Fronto-parietal \\
\hline 105 & -52 & 28 & 17 & VPFC & Fronto-parietal \\
\hline 106 & -44 & -63 & -7 & Occipital & Occipital \\
\hline 107 & 17 & -68 & 20 & Occipital & Occipital \\
\hline 108 & 36 & -60 & -8 & Occipital & Occipital \\
\hline 109 & -34 & -60 & -5 & Occipital & Occipital \\
\hline 110 & 39 & -71 & 13 & Occipital & Occipital \\
\hline 111 & 19 & -66 & -1 & Occipital & Occipital \\
\hline 112 & -16 & -76 & 33 & Occipital & Occipital \\
\hline 113 & 9 & -76 & 14 & Occipital & Occipital \\
\hline 114 & 15 & -77 & 32 & Occipital & Occipital \\
\hline 115 & 29 & -73 & 29 & Occipital & Occipital \\
\hline 116 & -29 & -75 & 28 & Occipital & Occipital \\
\hline 117 & 20 & -78 & -2 & Occipital & Occipital \\
\hline 118 & -18 & -50 & 1 & Occipital & Occipital \\
\hline 119 & -29 & -88 & 8 & Post occipital & Occipital \\
\hline 120 & 13 & -91 & 2 & Post occipital & Occipital \\
\hline 121 & 27 & -91 & 2 & Post occipital & Occipital \\
\hline 122 & -4 & -94 & 12 & Post occipital & Occipital \\
\hline 123 & -5 & -80 & 9 & Post occipital & Occipital \\
\hline 124 & 29 & -81 & 14 & Post occipital & Occipital \\
\hline 125 & 33 & -81 & -2 & Post occipital & Occipital \\
\hline 126 & -37 & -83 & -2 & Post occipital & Occipital \\
\hline 127 & 46 & -62 & 5 & Temporal & Occipital \\
\hline 128 & 60 & 8 & 34 & $\mathrm{dFC}$ & Sensorimotor \\
\hline 129 & 58 & 11 & 14 & Frontal & Sensorimotor \\
\hline 130 & 53 & -3 & 32 & Frontal & Sensorimotor \\
\hline 131 & -42 & -3 & 11 & Mid insula & Sensorimotor \\
\hline 132 & -36 & -12 & 15 & Mid insula & Sensorimotor \\
\hline 133 & 33 & -12 & 16 & Mid insula & Sensorimotor \\
\hline 134 & -26 & -8 & 54 & Parietal & Sensorimotor \\
\hline 135 & -47 & -18 & 50 & Parietal & Sensorimotor \\
\hline 136 & -38 & -15 & 59 & Parietal & Sensorimotor \\
\hline 137 & 46 & -20 & 45 & Parietal & Sensorimotor \\
\hline 138 & -55 & -22 & 38 & Parietal & Sensorimotor \\
\hline 139 & -38 & -27 & 60 & Parietal & Sensorimotor \\
\hline 140 & -24 & -30 & 64 & Parietal & Sensorimotor \\
\hline 141 & 41 & -23 & 55 & Parietal & Sensorimotor \\
\hline 142 & 18 & -27 & 62 & Parietal & Sensorimotor \\
\hline 143 & -47 & -12 & 36 & Parietal & Sensorimotor \\
\hline 144 & 42 & -24 & 17 & Post insula & Sensorimotor \\
\hline 145 & -41 & -31 & 48 & Post parietal & Sensorimotor \\
\hline 146 & 10 & 5 & 51 & Pre-SMA & Sensorimotor \\
\hline 147 & -54 & -22 & 22 & Precentral gyrus & Sensorimotor \\
\hline 148 & -54 & -9 & 23 & Precentral gyrus & Sensorimotor \\
\hline 149 & 44 & -11 & 38 & Precentral gyrus & Sensorimotor \\
\hline 150 & -44 & -6 & 49 & Precentral gyrus & Sensorimotor \\
\hline
\end{tabular}

\begin{tabular}{lcccll}
\hline ROI \# & $\boldsymbol{x}$ & $\boldsymbol{y}$ & $\boldsymbol{z}$ & Label & Network \\
\hline 151 & 46 & -8 & 24 & Precentral gyrus & Sensorimotor \\
152 & 58 & -3 & 17 & Precentral gyrus & Sensorimotor \\
153 & 0 & -1 & 52 & SMA & Sensorimotor \\
154 & 34 & -39 & 65 & Sup parietal & Sensorimotor \\
155 & -53 & -37 & 13 & Temporal & Sensorimotor \\
156 & -41 & -37 & 16 & Temporal & Sensorimotor \\
157 & 59 & -13 & 8 & Temporal & Sensorimotor \\
158 & -54 & -22 & 9 & Temporal & Sensorimotor \\
159 & 43 & 1 & 12 & vFC & Sensorimotor \\
160 & -55 & 7 & 23 & vFC & Sensorimotor \\
\hline
\end{tabular}

The ROIs were obtained from Dosenbach et al. (2010), and sorted by their affiliating networks. The $x, y$, and $z$ coordinates were given in MNI space. 

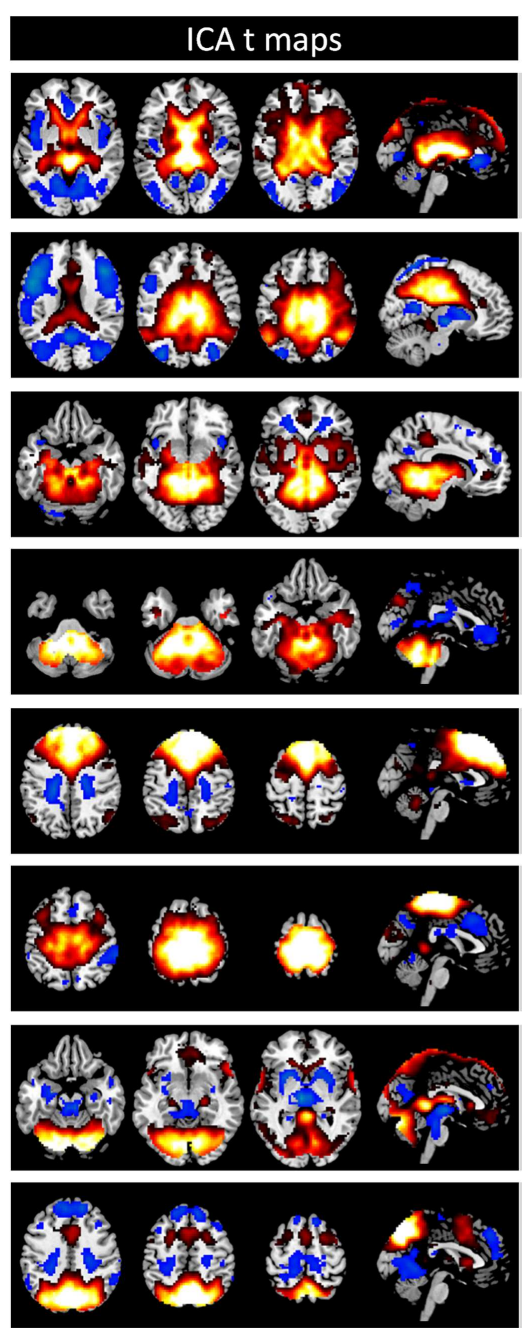

Negative $\square$
IC 4

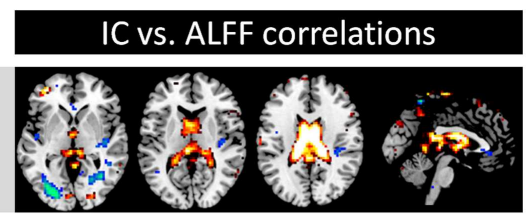

IC 5

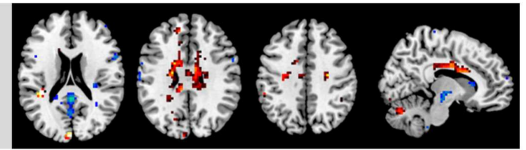

IC 6

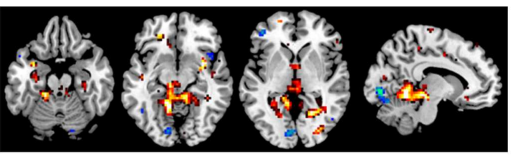

IC 8

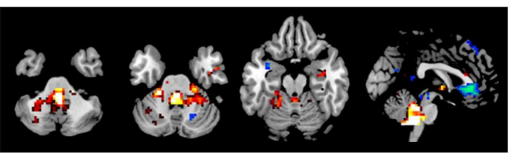

IC 10

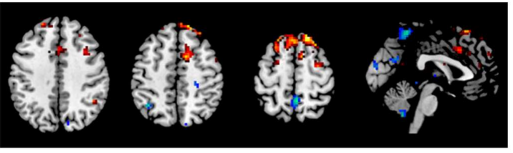

IC 17

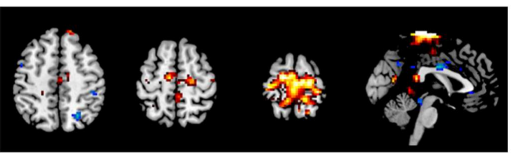

IC19

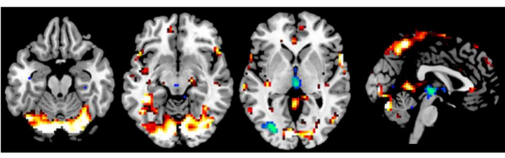

IC 20

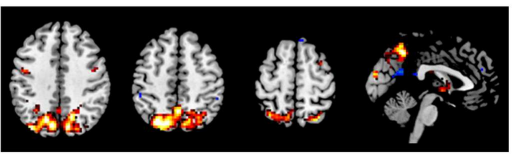

Positive
FIGURE A1 | Eight noise networks identified by spatial ICA (left column) and voxel-wise correlations between network strengths and ALFFs (right column). All maps are thresholded at $p<0.001$. For the ICA $t$ maps, display range is absolute $t$ value between 3.42 and 20 , and for the correlation maps, display range is absolute $r$ value between 0.364 and 0.6 . Hot and cold colors encode positive and negative effects, respectively. 